\title{
A regional Canadian expert consensus on recommendations for restoring exercise and pulmonary function testing in low and moderate-to-high community prevalence coronavirus disease 2019 (COVID-19) settings
}

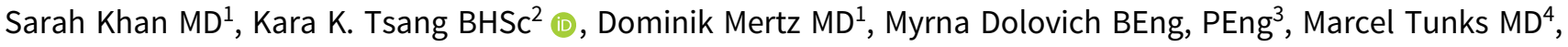 \\ Catherine Demers $\mathrm{MD}^{5}$, Kelly Hassall RRT, FCSRT, MEd ${ }^{6}$, Neil Maharaj MD${ }^{7}$, Karen Margallo ${ }^{8}$, Maureen Cividino MD ${ }^{9}$, \\ Zain Chagla MD ${ }^{10}$ and MyLinh Duong MBBS ${ }^{11}$ \\ ${ }^{1}$ Infection Prevention and Control, Hamilton Health Sciences, McMaster University, Hamilton, Ontario, Canada, ${ }^{2}$ Department of Biochemistry and Biomedical \\ Sciences, McMaster University, Hamilton, Ontario, Canada, ${ }^{3}$ Faculty of Health Sciences, Aerosol Research, McMaster University, Hamilton, Ontario, Canada, \\ ${ }^{4}$ Division of Respirology, St Joseph's Hospital, McMaster University, Hamilton, Ontario, Canada, ${ }^{5}$ Division of Cardiology and Health Evidence and Impact, \\ McMaster University, Hamilton, Ontario, Canada, ${ }^{6}$ Respiratory Therapy Department St Joseph's Healthcare Hamilton, Hamilton, Ontario, Canada, ${ }^{7}$ Michael G \\ DeGroote School of Medicine and Niagara Pulmonary Medicine \& Diagnostics, McMaster University, Hamilton, Ontario, Canada, ${ }^{8}$ Medical Diagnostic Unit, \\ Hamilton Health Sciences, McMaster University, Hamilton, Ontario, Canada, ${ }^{9}$ Occupational Health, St Joseph's Hospital, McMaster University, Hamilton, Ontario, \\ Canada, ${ }^{10}$ Infection Prevention and Control, St Joseph's Hospital, McMaster University, Hamilton, Ontario, Canada and ${ }^{11}$ Division of Respirology, Hamilton Health \\ Sciences, McMaster University, Hamilton, Ontario, Canada
}

To the Editor-Exercise and pulmonary function testing (PFT) are critical for the diagnosis, monitoring and management of cardiopulmonary disease. Previous respiratory pandemics have not impacted healthcare services to the same extent as coronavirus disease 2019 (COVID-19). Infection-specific guidance is now urgently needed to facilitate the resumption of pulmonary diagnostic services for low and moderate-to-high prevalence COVID-19 settings. Central to guidance development is the assessment of severe acute respiratory coronavirus virus 2 (SARS-CoV-2) transmission risk associated with these procedures, which are not recognized as aerosol-generating medical procedures (AGPs). However, they may carry risk due to the likelihood of generating coughs, exhaled respiratory droplets, and aerosols from the high ventilation and forced expiratory efforts. To date, there is no direct evidence for the risk of SARS-CoV-2 transmission in this context; thus, all guidelines are based on expert opinion from respiratory expert bodies. ${ }^{1-6}$ The aim of this document is to provide guidance for restoring exercise and pulmonary function testing in low and moderate-to-high prevalence settings of the COVID-19 pandemic. These recommendations are based on consensus from cardiopulmonary diagnostic service, occupational health, respiratory therapy, aerosol research, infection prevention and control (IPAC), and public health stakeholders to facilitate a uniform approach to the uptake and implementation of these recommendations. As further evidence emerges, revisions of these recommendations may be needed.

\section{Methods}

We undertook a literature review of guidelines and studies published in English between January 1, 2012, and September 30, 2020, on

Author for correspondence: Sarah Khan, E-mail: khan259@mcmaster.ca

Cite this article: Khan S, et al. (2021). A regional Canadian expert consensus on recommendations for restoring exercise and pulmonary function testing in low and moderate-to-high community prevalence coronavirus disease 2019 (COVID-19) settings. Infection Control \& Hospital Epidemiology, 42: 1535-1537, https://doi.org/ $10.1017 /$ ice.2020.1339 aerosol and droplet generation including risks for viral transmission during exercise testing and PFT. We communicated with respirology community members across Canada to ascertain the scope of and variation in conducting these procedures during the pandemic. We met with regional experts and stakeholders to discuss the available evidence on prescreening, aerosol and droplet generation, transmission risk, personal protective equipment (PPE) appropriateness, and infection control measures relevant to SARS-CoV-2. We developed recommendations for the restoration of exercise testing and PFT in low and moderate-to-high prevalence COVID-19 settings, defined by the rate of community transmission (excluding institutional outbreaks) below and exceeding 20 per 100,000 cases per week, respectively.

\section{Results}

Through direct communication with pulmonary diagnostic laboratories across Canada, we found wide variation in the delivery of these services during the pandemic and in the post-peak phase. We noted variable practices in screening procedures, COVID-19 testing, and PPE (procedural/surgical masks vs N95 respirators) across laboratories within and between institutions. We searched for international, ${ }^{2-4}$ national, ${ }^{1-5}$ and provincial $^{6}$ guidelines that provided recommendations for low or moderate-to-high community prevalence settings. Only 1 guideline provided recommendations for both settings. ${ }^{4}$ All were opinions from respiratory expert bodies and regarded these procedures as high risk for airborne infection transmission. As such, enhanced PPE and airborne precautions were recommended. No direct data were available regarding the risk of SARS-CoV-2 transmission related to pulmonary diagnostic procedures. Table 1 highlights the recommendations that were developed through consensus with stakeholders and after consideration of the limited available evidence.

\section{Discussion}

As cases of COVID-19 fluctuate, it is important to provide infection-specific guidance to facilitate the care of patients with cardiopulmonary disease. In our literature search, we found

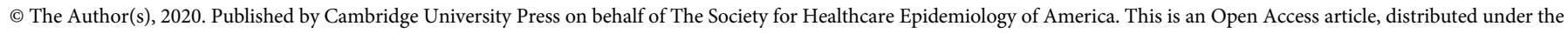
terms of the Creative Commons Attribution licence (http://creativecommons.org/licenses/by/4.0/), which permits unrestricted re-use, distribution, and reproduction in any medium, provided the original work is properly cited. 


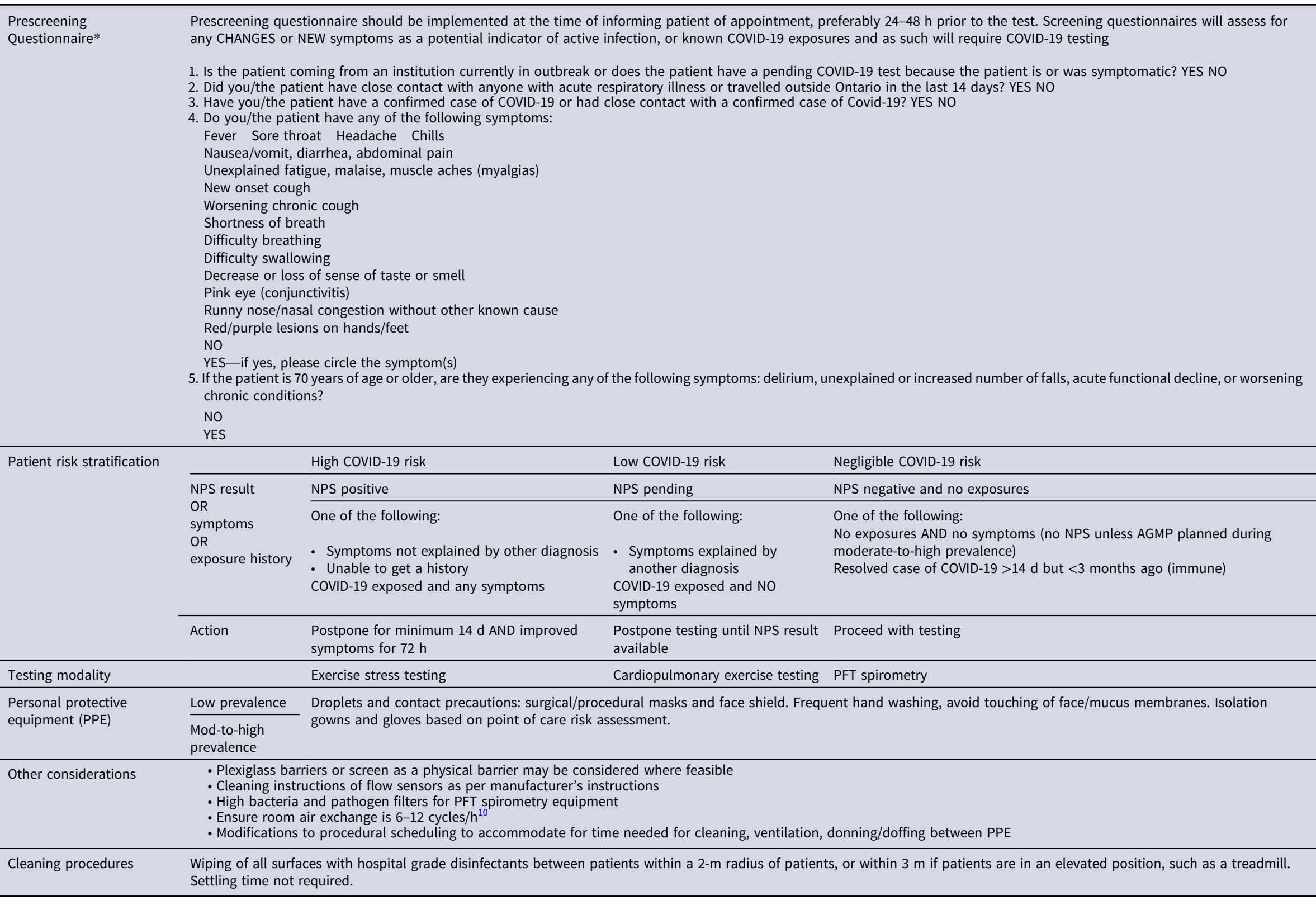

Note. PFT, pulmonary function testing; NPS, nasopharyngeal swabs for PCR detection of SARS-CoV-2. 
robust evidence supporting the generation of respiratory aerosols and droplets of varying sizes with coughing, sneezing, speech, singing, and breathing. ${ }^{7}$ There were no large or robust studies for the level of aerosols and respiratory droplets generated with spirometry or exercise testing. However, it is reasonable to assume that the deep exhalation and coughing associated with these pulmonary diagnostic procedures leads to aerosol and respiratory droplet generation. All previous guidelines have cautioned against these procedures as high-risk for airborne transmission and have recommended N95 respirators for protection. This recommendation is further supported by detectable SARS-CoV-2 viral RNA and viable virus within aerosoldroplet surrounding areas distant from infected patients, suggesting the potential for airborne transmission. ${ }^{8}$ However, no data to date have indicated that droplets carry sufficient viable virus to result in infection transmission; thus, it is not relevant in the healthcare setting. Furthermore, no strong data support meaningful reduction in transmission risk with use of N95 respirator instead of surgical masks for non-AGPs. For these reasons, we recommend the use of droplet and contact precautions with surgical or procedural masks and eye protection for these diagnostic procedures. If the point-of-care risk assessment suggests potentially elevated exposure risk, then isolation gowns and gloves may be added in a low-prevalence setting. In settings with low and moderate-to-high prevalence, pulmonary function testing should be deferred in patients who are risk-stratified as high risk for COVID-19.

Similar to other guidelines, we recommend that all facilities ensure that current recommended standards for heating, ventilation, and air conditioning are met, including temperature, humidity, and air changes. Environmental conditions likely play a role in cases in which aerosol transmission is the predominant mode of COVID-19 infection. ${ }^{9}$ Furthermore, under laboratory conditions, these environmental factors have been shown to determine the travel trajectory of expired particles. ${ }^{10}$ Lastly, universal masking and physical distancing should be maintained.

In conclusion, we developed COVID-19-specific recommendations for restoring exercise and PFT using a process of consensus involving all relevant key stakeholders becase there are no data available to inform the risk of SARS-CoV-2 transmission related to these pulmonary diagnostic procedures. Our guidance document incorporates a patient- and community-level risk-stratified approach that will facilitate the uniform adoption of IPAC practices across laboratories while protecting patients and staff in lowand high-prevalence settings.

\section{Acknowledgments.}

Financial support. No financial support was provided relevant to this article.

Conflicts of interest. All authors report no conflicts of interest relevant to this article.

\section{References}

1. Stanojevic S, Beaucage F, Comondore V, et al. Resumption of pulmonary function testing during the post-peak phase of the COVID-19 pandemic a position statement from the Canadian Thoracic Society and the Canadian Society of Respiratory Therapists. Canadian Thoracic Society website. $\quad$ https://cts-sct.ca/wp-content/uploads/2020/07/ CTS_CSRT_COVID_PFT_Final-July12_2020.pdf. Published 2020. Accessed Novmebr 20, 2020.

2. Wilson KC, Kaminsky DA, Michaud G, et al. Restoring pulmonary and sleep services as the COVID-19 pandemic lessens: from an association of pulmonary, critical care, and sleep division directors and American Thoracic Society-coordinated task force. Ann Am Thorac Soc 2020;17:1343-1351.

3. Singh SJ, Bolton C, Nolan C, et al. BTS Guidance for pulmonary rehabilitation-reopening services for the 'business as usual' participants. British Thoracic Society website. https:/www.brit-thoracic.org.uk/ document-library/quality-improvement/covid-19/pulmonary-rehabilitationreopening-services-for-the-business-as-usual-participants/. Published 2020. Accessed November 2020.

4. European Respiratory Society. Recommendation from ERS Group 9.1 (Respiratory function technologists /Scientists) lung function testing during COVID-19 pandemic and beyond. Black Lung Center of Excellence website. https://blacklungcoe.org/european-respiratory-societyers-recommendations-on-lung-function-testing-during-the-covid-19pandemic-and-beyond/. Published 2020. Accessed November 20, 2020.

5. Position statement on procedures creating a heightened risk of infection during an outbreak of a communicable respiratory disease. The Canadian Society of Respiratory Therapists website. https://www.csrt. com/wp-content/uploads/CSRT-Procedures-Duringan-Outbreak-April2020-v2.pdf. Published 2020. Accessed November 20, 2020.

6. Ontario Agency for Health Protection and Promotion (Public Health Ontario). IPAC Recommendations for Use of Personal Protective Equipment for Care of Individuals With Suspected or Confirmed COVID-19. Toronto: Queen's Printer for Ontario; 2020.

7. Anfinrud P, Stadnytskyi V, Bax CE, Bax A. Visualizing speech-generated oral fluid droplets with laser light scattering. $N$ Engl $J$ Med 2020;382:2061-2063.

8. Liu Y, Nong Z, Chen, Y, et al. Aerodynamic analysis of SARS-CoV-2 in two Wuhan hospitals. Nature 2020;582:557-560.

9. $\mathrm{Lu} \mathrm{J.} \mathrm{Gu} \mathrm{J,} \mathrm{Li} \mathrm{K,} \mathrm{et} \mathrm{al.} \mathrm{COVID-19} \mathrm{outbreak} \mathrm{associated} \mathrm{with} \mathrm{air} \mathrm{conditioning}$ in restaurant, Guangzhou, China, 2020. Emerg Infect Dis 2020;26: 1628-1631.

10. Guidelines for environmental infection control in health-care facilities. Appendix B. Air. Centers for Disease Control and Prevention website. https:/www.cdc.gov/infectioncontrol/guidelines/environmental/appendix/ air.html. Published 2003. Accessed September 28, 2020. 